September 9, 2000

BI-TP 2000/31

\title{
The Search for the QGP: A Critical Appraisal
}

\author{
Helmut Satz \\ Fakultät für Physik, Universität Bielefeld \\ D-33501 Bielefeld, Germany
}

\begin{abstract}
:
Over the past 15 years, an extensive program of high energy nuclear collisions at BNL and CERN was devoted to the experimental search for the quark-gluon plasma predicted by QCD. The start of RHIC this year will increase the highest available collision energy by a factor 10. This seems a good time for a critical assessment: what have we learned so far and what can we hope to learn in the coming years?
\end{abstract}

\footnotetext{
${ }^{1}$ Invited talk at Lattice 2000: XVIII International Symposium on Lattice Field Theory, August 17-22, 2000, Bangalore/India; Proceedings to appear in Nucl. Phys. B. Also presented at the RHIC and AGS Users Meeting, August 7-8, 2000, Brookhaven National Laboratory, Upton/NY, USA, and at Bologna 2000: Structure of the Nucleus at the Dawn of the Century, May 29-June 3, 2000, Bologna/Italy.
} 
"What's the good of Mercator's North Poles and Equators,

Tropics, Zones and Meridian Lines?"

So the Bellman would cry: and the crew would reply

"They are merely conventional signs!"

Lewis Carroll,

The Hunting of the Snark

\section{Expecting the Unexpected}

QCD predicts that at high energy density, hadronic matter will turn into a plasma of deconfined quarks and gluons. Eighteen years ago, theorists and experimentalists met in Bielefeld to chart the course for the study of quark-gluon plasma formation in heavy ion collisions. It was noted then: "In the past decades, we have investigated the strong interaction of elementary particles at higher and higher energies. So far, we have not been able to study in parallel strongly interacting macroscopic systems at higher and higher [energy] density. The thermodynamics of very dense matter is largely an unexplored field for terrestial experimental physics" [1].

In 1986/1987, experimental studies of high energy nuclear collisions began at the BNL-AGS and the CERN-SPS. The AGS started with ${ }^{28} \mathrm{Si}$ beams (at $15 \mathrm{GeV} /$ nucleon) and later went on to ${ }^{200} \mathrm{Au}$ beams (at $12 \mathrm{GeV} /$ nucleon) incident on heavy targets. At CERN, ${ }^{16} \mathrm{O}$ and ${ }^{32} \mathrm{~S}$ beams (at $200 \mathrm{GeV} /$ nucleon) were followed in 1995 by ${ }^{208} \mathrm{~Pb}$ beams (at $160 \mathrm{GeV} /$ nucleon). While both labs have by now provided a wealth of data on the hadronic final states produced in the different collisions, the AGS program did not include any experiment able to reach back into the early 'hot' stages of the collision evolution. Moreover, estimates in general indicate that with the AGS beam energy one can at best barely reach the initial energy densities required for quark-gluon plasma formation. For these reasons, most of our present knowledge about a possible onset of deconfinement in nuclear collisions has come from the CERN-SPS.

At the beginning, the search for colour deconfinement in high energy nuclear collisions was very much an exploratory endeavor. As in most explorations, the enthusiasm of the explorers was greater than their knowledge of what to expect or look for. There was a general feeling that if the quark-gluon plasma was indeed produced, it would manifest itself in a variety of unknown but dramatic ways, including the end of the world; this, however, was ruled out [2]. So the basic idea in the planning of experiments was to cover all possibilities, hoping that in this way the QGP would be observed somehow, somewhere, preferably everywhere.

Today, we are somewhat wiser. Changing conditions generally lead to a change in the related observables; if this happens in a monotonic way, it is indeed merely a conventional sign and has nothing to do with any phase transition. What we are really looking for is something like an 'order parameter' in statistical mechanics: an observable which remains constant over a range of different conditions, but which then at a certain point starts changing. Such non-analytic behaviour could be the consequence of a critical or transition point. It is not yet known if an experimentally accessible order parameter for deconfinement really exists. 
It is clear today, however, that hard and soft probes of nuclear collisions provide information about different stages of the produced state, determined by the scale of the probing phenomenon. For hard probes, the associated length scale is much less than the confinement scale $\Lambda_{\mathrm{QCD}}^{-1} \sim 1 \mathrm{fm}$; hence these can be used to study directly the shortdistance features of a hot deconfined medium. Soft probes are of hadronic size and appear when the density of the medium has dropped sufficiently to allow the existence of hadrons. Hence they naturally provide information about the hadronic state of the medium; about earlier states they can at best give indirect information.

The hadronisation of the QGP could lead to a hot interacting hadronic medium, or it could directly result in free-streaming hadrons and/or resonances. Hence there are three different stages to be considered: the deconfined medium (QGP), a possible interacting hadronic medium, and the final hadronic state.

Let us recall the situation at the beginning of the experimental QGP search some fifteen years ago. The main phenomena predicted to constitute direct hard probes of the QGP were:

- Hard thermal dilepton/photon emission [3, 4; their spectrum should serve as a thermometer of the thermal medium from which they were emitted.

- Jet quenching [5, 6]; the energy loss of fast partons passing through a deconfined medium should exceed that suffered in a passage through hadronic matter.

- $J / \psi$ suppression [7, 8]; colour screening in a deconfined medium leads to quarkonium dissociation, while tightly bound heavy quark states are not effected by a hadronic medium.

A possible interacting hadronic medium could be identified by

- in-medium modifications of resonances observable in their dilepton decays [9, 10]; in particular, changes of the $\rho$ mass or width should be observable in this decay pattern.

The proposed soft probes of the hadronic final state were:

- Strangeness enhancement [11, 12]; a hot QGP contains strange and the different nonstrange quark species in almost equal amounts, which, if preserved up to hadronization, should result in more abundant strangeness production than observed in $\mathrm{p}-\mathrm{p}$ interactions.

- Transverse momentum broadening and flow [13, 14]; compared to p-p interactions, a hot initial QGP could lead to more pronounced expansion and/or specific expansion patterns.

In addition, some more or less exotic states (strangelets, disoriented chiral condensates) or phenomena (softest point, tricritical point, event-by-event fluctuations) were proposed at various times as QGP or transition signatures; so far, none of these were observed.

Thermal dileptons and photons were the subject of intensive experimental studies. Nevertheless, there is up to now no real evidence for their production. The observed intermediate mass dilepton enhancement [15] may turn out to be a first indication of thermal dileptons [16], but so far its origin remains completely open. Moreover, real or virtual thermal photons, if observed, indicate the temperature of the medium, not its confinement status. 
Much interesting theoretical work on jet quenching has appeared in recent years (see [6] for a survey); but energy limitations put the effect out of reach for AGS and SPS. Hopefully it will hopefully enter as a viable probe with the advent of RHIC and LHC.

All other phenomena were indeed observed in high energy nuclear collisions. However, we shall see that all occur in fact already under conditions where presumably no QGP can be formed, e. g., in p-p or p-A interactions. Hence their observation as such cannot be taken as evidence for QGP production; it is crucial to specify if, when and how the observed behaviour constitutes an unconventional sign. Various studies over the past years have led to a systematic approach to this problem, and we shall here try to apply it to the different observed phenomena.

Before doing that, however, let us briefly consider the thermodynamic conditions necessary for QGP formation and compare them to what can be achieved in nuclear collisions. From finite temperature lattice QCD we know that deconfinement and chiral symmetry restoration occur in an equilibrated medium of vanishing baryon number density at a temperature of about $170-180 \mathrm{MeV}$; hence energy densities of some $1-3 \mathrm{GeV} / \mathrm{fm}^{3}$ are required for QGP formation [17]. Percolation theory provides estimates valid also in non-equilibrium conditions; they lead to similar values [18].

We want to compare the predicted critical conditions to those obtained in nuclear collisions. The well-known evolution based on freely streaming hadrons [19] leads to the initial energy density

$$
\epsilon_{0}=\frac{1}{\tau_{0} A_{T}}\left(\frac{d E}{d y}\right)_{y=0},
$$

where $A_{T}$ denotes the transverse overlap area of the two colliding nuclei, $\tau_{0} \simeq 1 \mathrm{fm}$ the formation time of the initial medium, and $(d E / d y)_{y=0}$ the measured total energy of all the hadrons emitted at central rapidity. Alternatively, $\epsilon_{0}$ can be expressed in terms of the measured central multiplicity $(d N / d y)_{y=0}$ and the average energy $p_{0}$ of the hadrons at $y=0$, with $(d E / d y)_{y=0}=p_{0}(d N / d y)_{y=0}$. The relevant quantities in $\mathrm{Pb}-\mathrm{Pb}$ collisions were determined by several SPS groups [20, 21]. In Fig. 1, we show the resulting estimate of the initial energy density in SPS Pb-Pb collisions as function of the number $N_{w}$ of participating nucleons. Through a Glauber analysis, this can be related to the impact parameter of the collision [21, 22, 23]. We conclude that these collisions do reach the regime in which deconfinement could be expected in the early stages of the produced medium.

\section{Charmonium Suppression}

At present, the only accesible hard probe for a direct test of the confinement status of the early medium is charmonium production. The binding energy of the $J / \psi$ is about $3 \Lambda_{\mathrm{QCD}}$ and its radius about $0.2 \mathrm{fm}$, so that it indeed 'sees' the short scales of a hot prehadronic medium. In a deconfining environment, it dissolves once the screening radius falls below its binding radius [0]. On a microscopic level, short-distance QCD calculations show that $J / \psi$-hadron collisions at the hadron momenta attained in SPS collisions do not lead to significant dissociation [8]. Gluons confined to hadrons are not sufficiently hard for a break-up; deconfinement hardens the gluons and allows charmonium dissociation. Hadronic models claiming a large $J / \psi$ dissociation cross section in the threshold region [24] are not in accord with data from $J / \psi$ photoproduction [25]. 


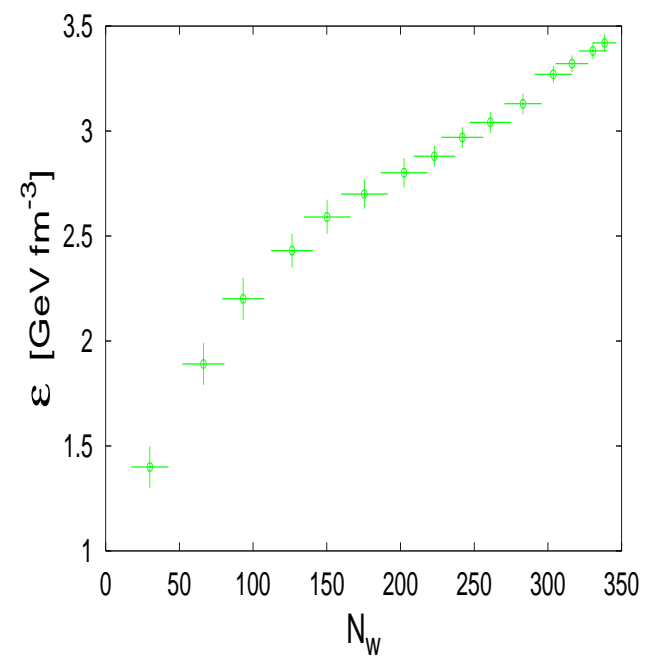

Figure 1: The variation of the initial energy density with the number of participant nucleons [21, 23].

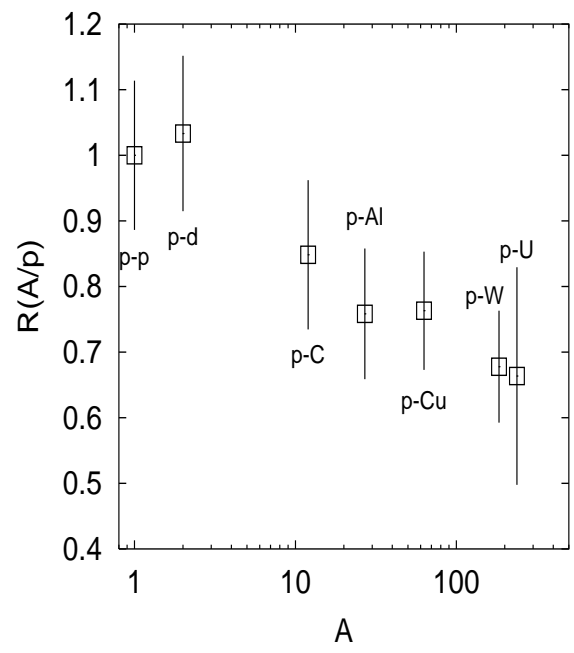

Figure 2: $J / \psi$ suppression in p-A collisions [29].

The suppression of $J / \psi$ production was observed quite early in the CERN heavy ion program [26]; but it was soon noted that it occurs as well already in p-A collisions (see Fig. 2), where it inceases with increasing A [27]-29]. It thus depends on the presence of a bulk medium, the nucleus; but the nucleus clearly is a confined medium and hence $J / \psi$ suppression cannot generally mean deconfinement 30.

Before one can apply $J / \psi$ suppression as probe in nuclear collisions, it is therefore necessary to carry out a sequence of steps:

- study the elementary production process in p-p collisions to determine its behaviour in the absence of any bulk medium,

- determine its behaviour in the confined nuclear medium given by p-A collisions;

- then check if in A-B collisions there are deviations from the 'normal' behaviour observed in the confined nuclear medium.

It should be emphasized that this procedure was by no means evident from the beginning; it is a lesson in method learned in over ten years of extensive experimental $J / \psi$ production studies and the corresponding theoretical attempts to understand the results (see 31, 32]). I want to suggest here that it in fact defines a more general approach which any experimental QGP probe must follow in order to become conclusive.

$J / \psi$ production in high energy nucleon-nucleon collisions starts with the fusion of a target and a projectile gluon to form a colour octet $c \bar{c}$ pair. In the colour field of the interaction region, the pair neutralizes its colour to form a colour singlet state of $J / \psi$ quantum numbers. While the $c \bar{c}$ production can be described perturbatively, the colour neutralization is of non-perturbative nature. It can be described conceptually by the colour evaporation model [33], which is based on the total sub-threshold charm cross section. This is obtained by integrating the perturbatively calculated $c \bar{c}$ production over 
the mass interval from $2 m_{c}$ to $2 m_{D}$,

$$
\sigma_{c \bar{c}}(s)=\int_{2 m_{c}}^{2 m_{D}} d \hat{s} \int d x_{1} d x_{2} g_{p}\left(x_{1}\right) g_{t}\left(x_{2}\right) \sigma(\hat{s}) \delta\left(\hat{s}-x_{1} x_{2} s\right),
$$

where $g_{p}(x)$ and $g_{t}(x)$ denote the gluon densities, $x_{p}$ and $x_{t}$ the fractional momenta of projectile and target gluons, and $\sigma$ the $g g \rightarrow c \bar{c}$ cross section. The colour evaporation model now states that the production cross section of any charmonium state $i$ is given by

$$
\sigma_{i}(s)=f_{i} \sigma_{c \bar{c}}(s)
$$

where $f_{i}$ is an energy-independent constant to be determined empirically. This has two clear consequences: the energy dependence of any charmonium production cross section is predicted to be that of the perturbatively calculable sub-threshold charm cross section, and the production ratios of different charmonium states must be energy-independent. Both sets of predictions agree very well with data over a considerable energy range. As illustration, we show in Fig. 3 that the energy dependence is correctly described for the $J / \psi$, as it is in a corresponding treatment of $\Upsilon$ production, where data are available up to $1.8 \mathrm{TeV}$. For further studies, see [33].
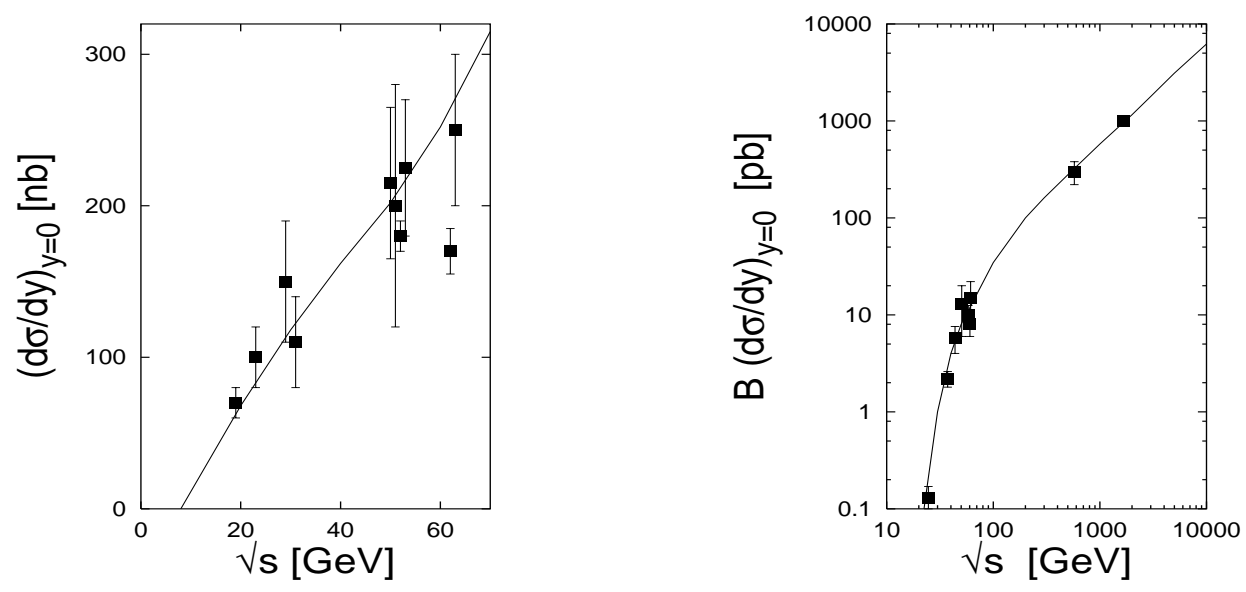

Figure 3: The energy dependence of $J / \psi$ hadroproduction (left) and of $\Upsilon$ hadroproduction (right) based on the colour evaporation model using MRS D-' parton distributions [33].

While the colour evaporation model gives an empirically well supported description of charmonium and bottonium production, it does not provide a space-time description of the production process. For charmonium production in $\mathrm{p}-\mathrm{A}$ and $\mathrm{A}-\mathrm{B}$ collisions, this is crucial, however, and hence a more detailed model for colour neutralization is needed. The colour octet model [34] proposes that the colour octet $c \bar{c}$ combines with a soft collinear gluon to form a singlet $(c \bar{c}-g)$ state. After a short relaxation time $\tau_{8}$, this pre-resonant charmonium state (a 'higher Fock space component' of the $J / \psi$ ) turns into the physical $c \bar{c}$ singlet $J / \psi$ mode (the 'basic Fock space component') by absorbing the accompanying gluon. Such a formation process occurs as well for $\chi_{c}$ and $\psi^{\prime}$ production. The colour octet model encounters difficulties if the collinear gluons are treated perturbatively, illustrating once more that colour neutralization seems to require non-perturbative elements [35]. However, it does provide a conceptual basis for the evolution of the formation process. 
The life-time $\tau_{8}$ and the size $r_{8}$ of pre-resonant charmonium can be estimated 36. Both are essentially determined by the lowest momentum possible for confined gluons and hence are the same for the different states $J / \psi, \chi_{c}$ and $\psi^{\prime}$, with $r_{8} \simeq \tau_{8} \simeq 0.20-0.25$ $\mathrm{fm}$. This has striking consequences for charmonium production in $\mathrm{p}-\mathrm{A}$ collisions.

$J / \psi$ production in p-A collisions has been studied in fixed target experiments with incident protons of momenta between 200 and $800 \mathrm{GeV} / \mathrm{c}$ [27]-[29]. Data are taken mainly for $x_{F}>0$, which gives the nascent $J / \psi$ momenta of $30 \mathrm{GeV} / \mathrm{c}$ or more in the target rest frame. As a result, the transition $(c \bar{c}-g) \rightarrow J / \psi, \chi$ or $\psi^{\prime}$ occurs at the edge of the target nucleus or completely outside; the nuclear medium of the target sees only the passage of pre-resonance states. Since these have essentially the same size for all charmonia, the observed attenuation of the production rates should be the same for $J / \psi$ as for $\psi^{\prime}$. This is indeed found to be quite well satisfied, as shown in Fig. U [29. Earlier attempts to explain charmonium suppression in $\mathrm{p}$-A interactions in terms of the absorption of physical $J / \psi$ states [30] had encountered difficulties precisely because of this feature. The equal attenuation of $J / \psi$ and $\psi^{\prime}$ for $x_{F}>0$ is a natural consequence of pre-resonance absorption; it can never be obtained for the physical $J / \psi$ and $\psi^{\prime}$ states with their very different geometric sizes.

The cross section for the absorption of pre-resonance charmonium in nuclear matter can be determined through a Glauber analysis of p-A data. Such an analysis of the most recent data for 200 and $450 \mathrm{GeV}$ data [29] gives $\sigma_{c \bar{c} g-N}=6.9 \pm 1.1 \mathrm{mb}$, in accord with previous analyses [22] and theoretical estimates [36].

With this, the first two steps of the mentioned procedure for the application of $J / \psi$ suppression as deconfinement probe are completed. We have a viable theoretical description of the elementary production process, although some aspects of colour neutralization still remain unclear [35]. We know how this process is modified in the confined medium provided by nuclear matter. The expected 'normal' behaviour is clear, and the probe can now be applied to nuclear collisions in order to look for an onset of deconfinement. In Fig. 5, the production rates measured in different $\mathrm{p}-\mathrm{A}$ and centrality-integrated A-B collisions with light ion beams 29 are seen to agree completely with 'normal' pre-resonance suppression; this agreement persists as well for the centrality dependence of $J / \psi$ production in $\mathrm{S}-\mathrm{U}$ interactions, as determined by measuring the associated transverse energy $E_{T}[22$. Hence up to central S-U collisions, there is no unconventional sign.

Before turning to $\mathrm{Pb}-\mathrm{Pb}$ collisions, we note a characteristic feature of $J / \psi$ suppression by deconfinement. The $J / \psi$ 's measured in hadronic collisions originate from three different sources: about $60 \%$ are directly produced $J / \psi$ 's, while about $32 \%$ arise from $\chi_{c}$ decay and the remaining $8 \%$ from $\psi^{\prime}$ decay. Since the decay widths are extremely small, the decays occur far outside the interaction region; any produced medium therefore effects (after the pre-resonance stage) the survival of three different charmonium states. Dissociation calculations based on a screened confining potential 37] indicate that the $\chi_{c}$ and the $\psi^{\prime}$ are dissociated essentially at the deconfinement point, while the more tightly bound $J / \psi$ state requires higher energy densities to 'melt'. This result is confirmed by collision calculations based on the different binding energies of the three states [8]; however, here one finds that the $\psi^{\prime}$, which is only $60 \mathrm{MeV}$ below the open charm threshold, can also be dissociated by any interaction in a confined medium. These considerations predict a sequential suppression pattern 38, 39]: first any medium, whether confined or deconfined, will lead to $\psi^{\prime}$ suppression. When the energy density is increased beyond the deconfine- 


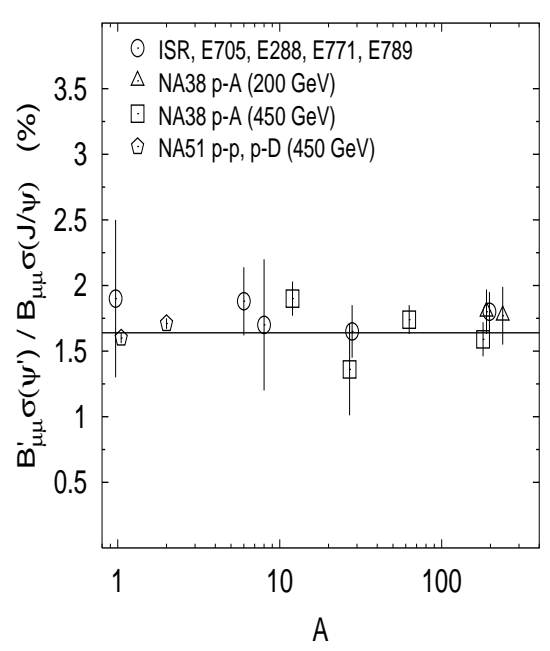

Figure 4: The relative A-dependence of $J / \psi$ and $\psi^{\prime}$ production in $\mathrm{p}-\mathrm{A}$ collisions [26].

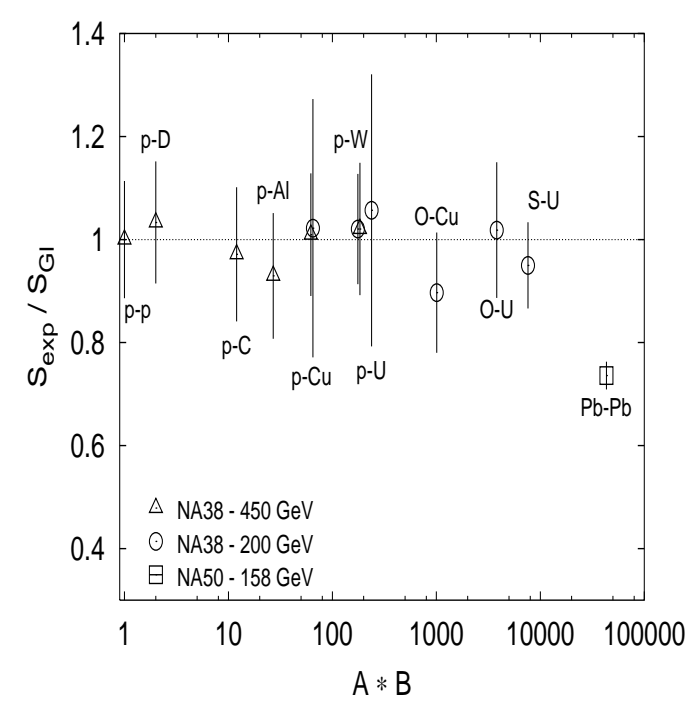

Figure 5: Centrality integrated rates for $J / \psi$ production in $A-B$ collisions, normalized to pre-resonance absorption in nuclear matter [29, 21].

ment point $\epsilon_{c}, \chi_{c}$ suppression will set in, and beyond $1.5-2 \epsilon_{c}$, also the directly produced $J / \psi$ 's will be suppressed.

$J / \psi$ suppression by colour deconfinement is thus predicted to have two specific qualitative features: apart from the small contribution due to $\psi^{\prime}$ decay, it should set in quite suddenly, since we are dealing with critical behaviour, and it should show a characteristic two-step pattern, corresponding to sequential $\chi_{c}$ and $J / \psi$ suppression.

The experimental studies over the past four years 21] have shown that in $\mathrm{Pb}-\mathrm{Pb}$ interactions, $J / \psi$ production is reduced beyond the expected pre-resonance absorption (see Fig. 5). In detail, this anomalous suppression shows the following features:

- In peripheral $\mathrm{Pb}-\mathrm{Pb}$ collisions, $J / \psi$ production follows the normal pattern of preresonance suppression up to $E_{T} \simeq 40 \mathrm{GeV}$.

- With increasing centrality, the anomalous suppression sets in quite suddenly at $E_{T} \simeq 40 \mathrm{GeV}$, corresponding to $N_{w} \simeq 100$ participating nucleons.

- At $E_{T} \simeq 100 \mathrm{GeV}$, a second drop of $J / \psi$ production occurs; this corresponds to $N_{w} \simeq 300$ participating nucleons.

In Fig. 6, the result is shown as function of the associated transverse energy $E_{T}$, together with the expected form for pre-resonance absorption. Using a zero-degree calorimeter, the experiment also determines the number $N_{w}$ of participant nucleons for each value of $E_{T}$, as noted above for the threshold points. In Fig. 7, the $J / \psi$ survival probability in the produced medium, i.e., the production rate divided by the pre-resonance absorption, is shown as function of $N_{w}$, for all p-A and A-B data taken by the collaboration. Using the free-streaming evolution model [19], one can convert $E_{T}$ into energy density for the different reactions. This is shown in Fig. 8 [21], with the first drop occurring just above $2 \mathrm{GeV} / \mathrm{fm}^{3}$ and the second around $3 \mathrm{GeV} / \mathrm{fm}^{3}$. 


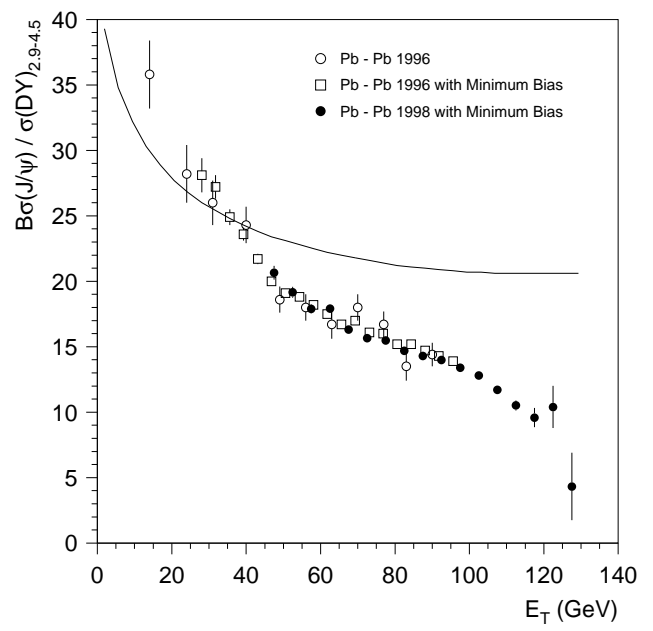

Figure 6: The $E_{T}$-dependence of the ratio $J / \psi /$ Drell-Yan production, compared to the normal dependence due to pre-resonance suppression [29, 21].

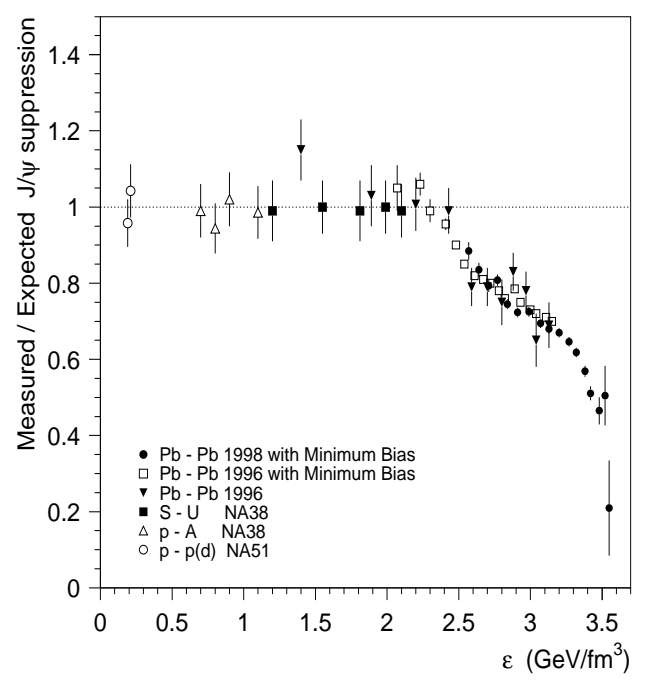

Figure 8: $J / \psi$ production in $A-B$ collisions, normalized to pre-resonance absorption in nuclear matter, as function of the initial energy density [21].

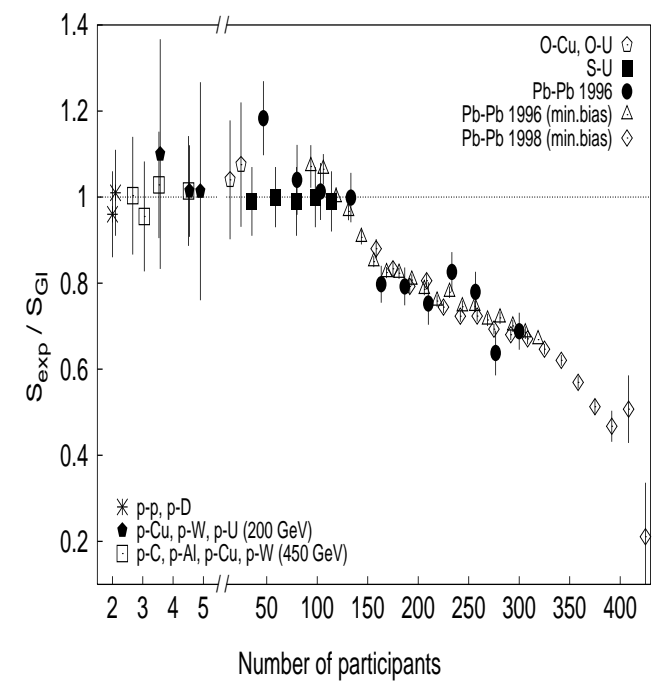

Figure 7: $J / \psi$ production in A-B collisions, normalized to pre-resonance absorption in nuclear matter, as function of the number of participating nucleons 21, 23.

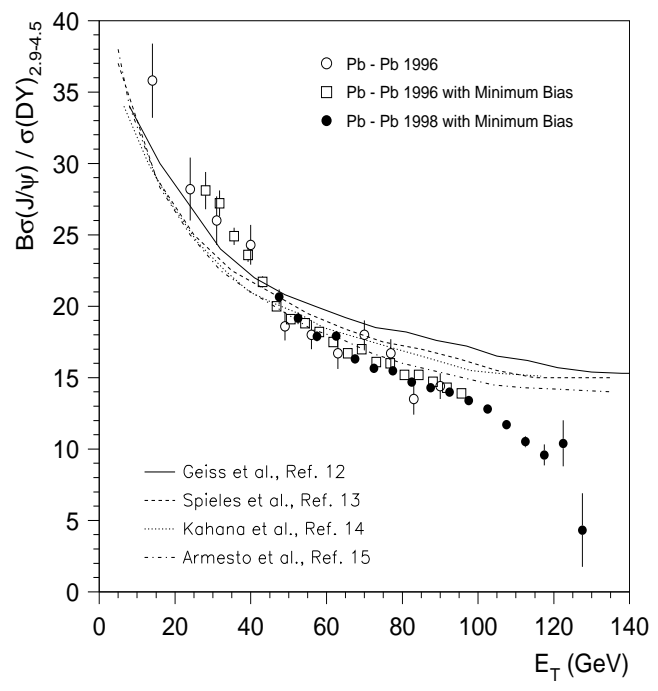

Figure 9: The $E_{T}$-dependence of the ratio $J / \psi /$ Drell-Yan production, compared to different hadronic comover models, with references as given in 29. 
The observed two-step form of anomalous suppression is thus the one predicted by deconfinement [38, 39]. In contrast, it is in qualitative and quantitative disagreement wit all hadronic comover models, as illustrated in Fig. 9. We conclude that the $J / \psi$ suppression pattern observed by the NA50 collaboration indeed constitutes an unconventional sign, which can be quite naturally interpreted as the onset of deconfinement in nuclear collisions [21].

However, this conclusion is still accompanied by a number of open questions which have to be answered in order to fully understand what is happening.

- What is the relevant variable which determines the onset of deconfinement - is it indeed the energy density, as suggested in Fig. 86? To answer this conclusively, experiments with different nuclei are needed.

- What is the origin of the rather steep final drop? The onset of suppression for direct production does not reproduce it in the observed strength [41]. Transverse energy fluctuations could form another or additional mechanism [42].

- How does the $\psi^{\prime}$ fit into the picture? As expected, it shows the same pre-resonance suppression as the $J / \psi$. In all $\mathrm{S}-\mathrm{U}$ collisions, however, it is significantly suppressed, with a rather abrupt onset at quite peripheral interactions. Because of the small binding energy, the $\psi^{\prime}$ could be dissociated by hadronic or partonic comovers.

- How do the suppression patterns for the different charmonium states vary with their transverse momentum? Here a normal and an anomalous behaviour can also be defined [43]; present data are still inconclusive.

- So far, charmonium production was studied experimentally with Drell-Yan production as reference. In view of the observed suppression of bound $c \bar{c}$ states in nuclear collisions, the unsuppressed behaviour $\left(\sim A^{2}\right)$ for unbound (open charm) $c \bar{c}$ production should be verified. In any case, open charm production provides a better reference, since it involves the same gluon distribution functions as charmonium production.

Some of these questions could well still be addressed by the SPS. The scaling variable, as noted, can be clarified by $\mathrm{A}-\mathrm{A}$ experiments at lower $\mathrm{A}$ than $\mathrm{Pb}$. To identify the origin of the second drop requires either experiments at lower A (SPS) or at higher incident energy (RHIC): does the drop shift to lower $N_{w}$ or higher $E_{T}$, respectively, as predicted if transverse energy fluctuations are responsible? In any case, a confirmation of the observed $J / \psi$ suppression pattern by RHIC, perhaps with even more pronounced thresholds, seems of basic importance for the entire QGP study. Finally we note that a recent study [44] invokes final state reinteractions to predict for A-A collisions (normalized to A) at very high energies a $J / \psi$ enhancement rather than suppression relative to p-p production rates. Similar conclusions are obtained through thermal colour neutralisation [45], although this model is not compatible with preliminary data on $\psi^{\prime} / \mathrm{J} / \psi$ production in $\mathrm{Pb}-\mathrm{Pb}$, compared to $\mathrm{S}-\mathrm{U}$ 40, 46]. The first $J / \psi$ data from RHIC, even if of low statistics, will thus already provide some interesting conclusions.

\section{In-Medium Hadron Modifications}

High energy nuclear collisions eventually produce a hadronic state, and it is clearly of interest to check if these hadrons form at some stage a dense, interacting medium. 
The $\psi^{\prime}$ suppression observed in S-U collisions could well be an indication for this, since the produced medium here does not effect $J / \psi$ production. A direct probe to study this question is provided by dilepton spectra in the hadronic resonance mass range. The decay width of the $\rho$ into a lepton pair gives this resonance a life-time of about $1 \mathrm{fm}$, so that any in-medium modifications should show up in the measurable dilepton spectrum [3, 4]. Most striking would be mass changes in the vicinity of the chiral symmmetry restoration point [47]; it is clear, however, that an interacting medium as such can quite generally lead to modified resonance properties 48.

The dilepton spectrum from very low masses up to the $J / \psi$ was measured by several SPS experiments [15, 49, 50]. In Fig. 10 it is seen that the spectrum measured for proton beams incident on both light and heavy targets is accounted for quite well by the decay of the known and observed hadronic states decaying into lepton pairs. For $\mathrm{S}-\mathrm{U}$ and $\mathrm{Pb}-\mathrm{Pb}$ collisions this is no longer the case; an excess is observed both for low mass pairs, below the $\rho-\omega$ peak, and for intermediate mass pairs, between $\phi$ and $J / \psi$ (see Fig. 11).
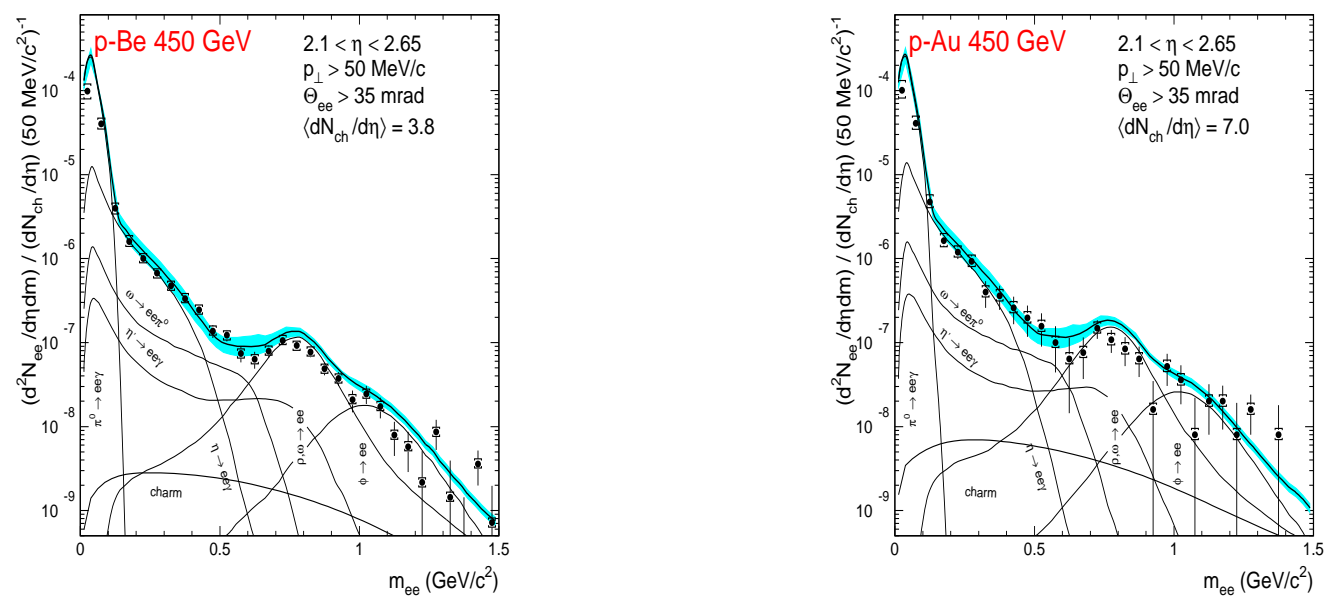

Figure 10: The dilepton spectrum in p-Be (left) and p-Au (right) collisions, compared to the combined results (band) of hadronic decay contributions [49.

The low mass enhancement has been the subject of much theoretical attention. A decrease of the $\rho$ mass in a hot medium near chiral symmetry restoration could indeed lead to such an effect 477; however, lattice studies do not show any evidence of a changed rho mass over a significant temperature range. On the other hand, in-medium broadening of the resonance due to hadronic interactions can as well reproduce the observed enhancement 48]. Hence it is at present not clear if the observed modifications of the dilepton spectrum indeed constitute an unconventional sign. The rather low signal-to-background ratio of present data moreover does not allow a very quantitative study of the effect. It is thus not clear yet if there is really a low mass enhancement and a distinct separate enhancement at higher masses, or if the two observations are one effect. So far, there is also no evidence of any onset or threshold. Some interesting recent work [16 has tried to interpret in particular the higher mass enhancement in terms of thermal dileptons. In summary, here more precise experimental data seem very necessary before any conclusion can be reached. 


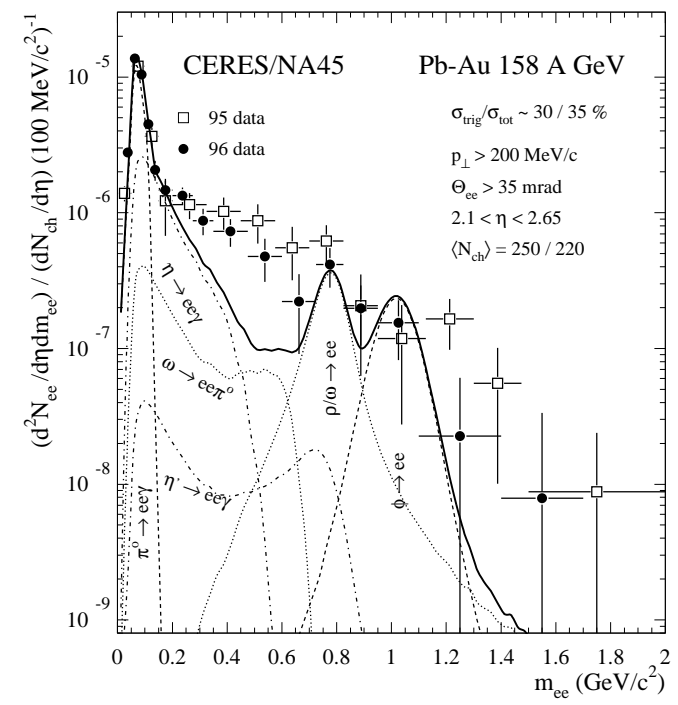

Figure 11: The dilepton spectrum in $\mathrm{Pb}-\mathrm{Pb}$ collisions, compared to the combined results (band) of hadronic decay contributions 49 .

\section{Strangeness and Thermalization}

One of the most remarkable observations in high energy multihadron production by whatever means is that the relative abundances of the secondary hadrons seem to be quite well described by an ideal resonance gas of a temperature $T_{h} \simeq 170 \mathrm{MeV}$ [51, 52]. The only and systematic exception is due to strange particles: in $\mathrm{p}-\mathrm{p}, \mathrm{p}-\overline{\mathrm{p}}$ and $e^{+} e^{-}$ collisions, these are produced less abundantly than expected in a resonance gas scheme. This reduction can be accounted for by a general strangeness suppression factor $\gamma_{s} \simeq 1 / 2$ : for each strange or antistrange quark in the hadron in question, one power of $\gamma_{s}$ reduces the production abundance. Thus the entire relative production spectrum of sometimes up to 30 different resonances can be described in terms of two parameters, $T_{h} \simeq 170 \mathrm{MeV}$ and $\gamma_{s} \simeq 1 / 2$ [52, as illustrated in Fig. 12.

The origin of such a "thermal" production scheme and of the observed strangeness suppression is not at all clear. Since the description is even valid in $e^{+} e^{-}$interactions with very few hadrons per unit rapidity, it can definitely not be caused by final state hadron interactions. Conceptually the most convincing picture is that any high energy collision implies a passage of colour charges through the vacuum, which is excited by this passage and subsequently neutralizes the excitation by a statistically governed formation of quark-antiquark pairs and gluons. As the bubble of excited vacuum expands, the quarks and gluons have to combine to produce the observed secondary hadrons, which reflect their "statistical" origin and the universal hadronization temperature $T_{h}$ [53]. While the hadronization temperature is universal, the energy density of the initial excited vacuum bubble will increase with increasing collision energy, and it will presumably also be higher in A-A than in p-p collisions at the same incident beam energy. A possible cause of the observed strangeness suppression is thus the higher strange quark mass, which makes it more difficult to form $s \bar{s}$ pairs than pairs of light $u$ or $d$ quarks. For an ideal quark gas, 


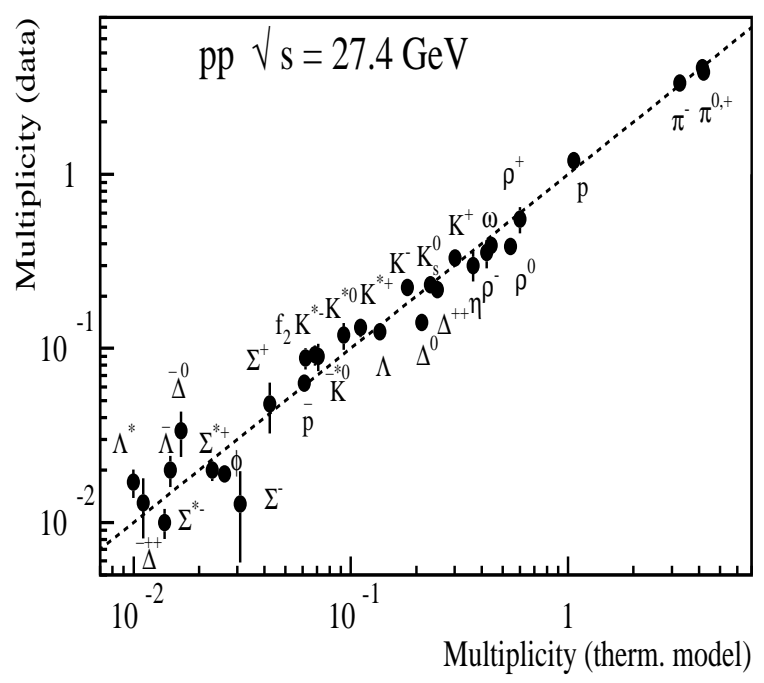

Figure 12: Hadron abundances for p-p collisions, compared to the prediction of an ideal resonance gas at $T_{h}=170 \mathrm{MeV}$ and $\gamma_{s}=0.5$ [52].

one would expect

$$
\gamma_{s}=1-\frac{m_{s}^{2}}{2 T_{0}^{2}}
$$

where $T_{0} \geq T_{h}$ is the initial temperature of the excited vacuum. An immediate prediction of this scheme is a diminishing of strangeness suppression in $\mathrm{p}-\mathrm{p}$ (or $\mathrm{p}-\overline{\mathrm{p}})$ collisions, either with increasing incident energy or at fixed energy with the number of produced secondaries per unit rapidity (i.e., with the energy deposit due to the collision). In Fig. 13, this is indeed observed, since in both cases the ratio of kaons to pions increases considerably, although the hadronization temperature $T_{h}$ determined through multiplicity integrated abundances remains the same.

For nucleus-nucleus collisions, a strangeness enhancement from the hadronic $\gamma_{s} \simeq 1 / 2$ towards $\gamma_{s}=1$ was predicted as consequence of colour deconfinement [11, 12]. In a sufficiently hot QGP, the higher strange quark mass would become less and less important, so that $u, d$ and $s$ quarks should eventually appear in equal abundance. If the cooling of the QGP prodeeded rapidly enough to preserve this relative abundance, the final hadronic state obtained from a hadronized QGP in nucleus-nucleus collisions should contain more strange hadrons than that formed in p-p interactions, i.e, without an initial QGP. In Fig. 14 we see that $\mathrm{Pb}-\mathrm{Pb}$ collisions at the SPS indeed lead to enhanced strangeness production, as again reflected in an increasing kaon to pion ratio. In Fig. 15, it is seen that the resonance gas description remains valid for centrality-integrated abundances, with the same $T_{h}$ as above, but with $\gamma_{s}$ close to unity.

Another possible origin for the strangeness suppression observed in $e^{+} e^{-}, \mathrm{p}-\mathrm{p}$ and $\mathrm{p}-\overline{\mathrm{p}}$ interactions could be the relatively small number of strange particles produced. The ideal resonance gas in its usual grand canonical formulation includes strangeness conservation on the average. If only one kaon and its antiparticle are produced in the event, strangeness conservation must be imposed exactly, and this leads to a strong appearent suppression compared to grand canonical rates [54, 55]. To illustrate: in the grand canonical formulation, a kaon acquires a Boltzmann factor $\exp \left\{-m_{K} / T_{h}\right\}$, while exact strangeness 

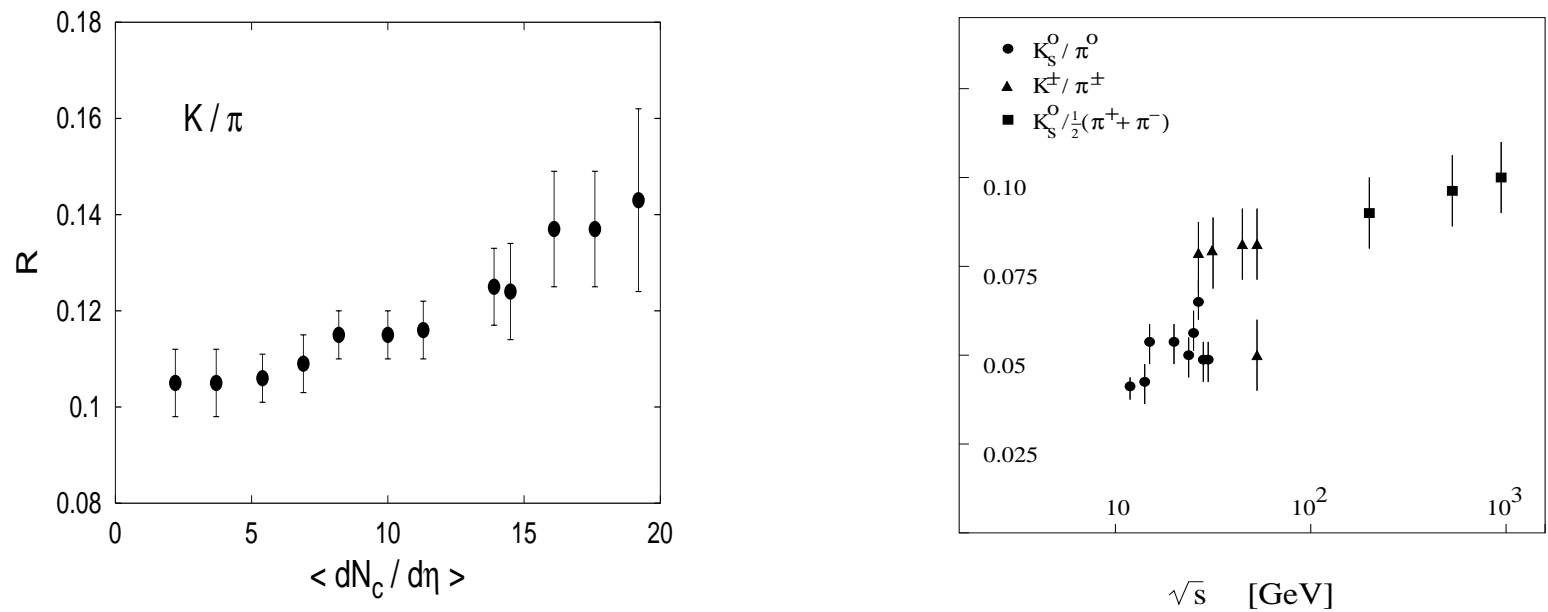

Figure 13: The ratio of kaon to pion production in $\mathrm{p}-\overline{\mathrm{p}}$ collisions (left) as function of the associated multiplicity [59] and (right) as function of the incident energy [57].

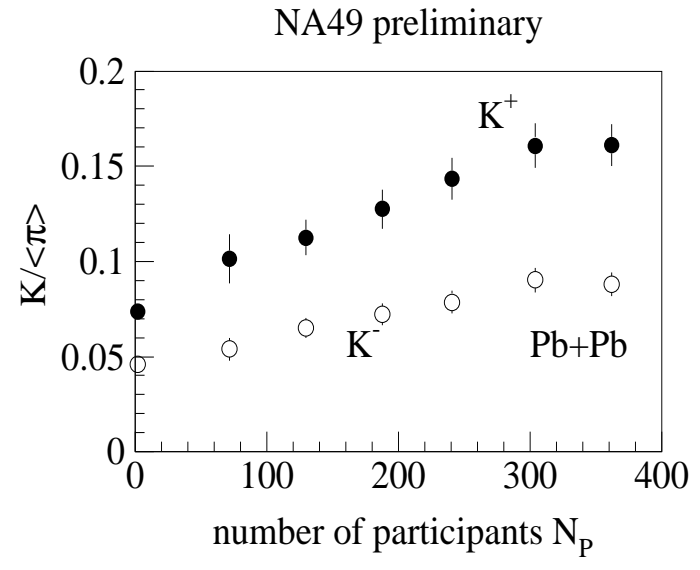

Figure 14: The ratio of kaon to pion production in $\mathrm{Pb}-\mathrm{Pb}$ collisions [58].

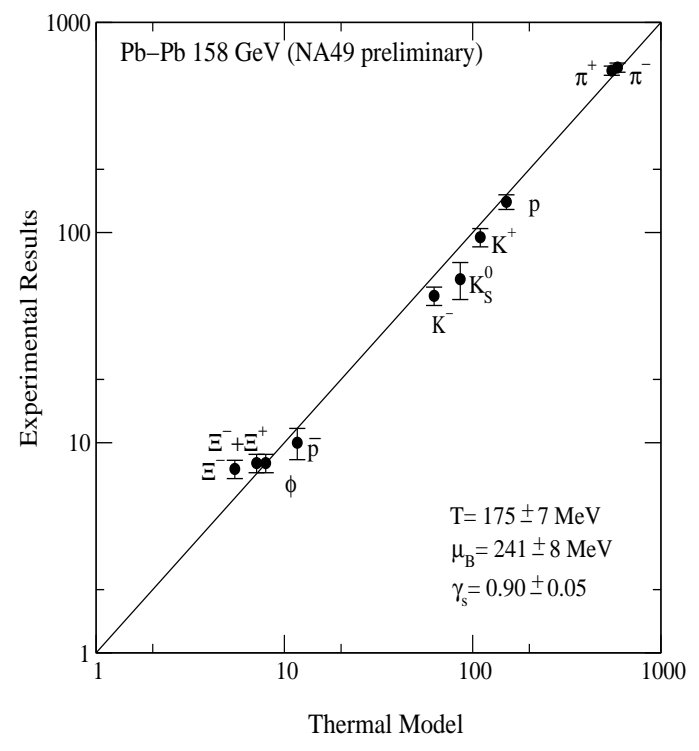

Figure 15: Hadron abundances for $\mathrm{Pb}-$ $\mathrm{Pb}$ collisions, compared to the prediction of an ideal resonance gas [62]. 
conservation reduces this to $\exp \left\{-2 m_{K} / T_{h}\right\}$, since the production of one kaon requires that of one antikaon (we neglect associated production for the sake of argument). These considerations lead to a strangeness suppression which is strong at low relative multiplicities and vanishes for large multiplicities when the grand canonical limit is reached [55]. The resulting suppression form is quite similiar to the pattern observed in the production of strange baryons and antibaryons, ranging from "suppression" in p-p and p-A to an "enhancement" in nuclear collisions [55].

In summary: since strangeness enhancement is observed already in hadron-hadron collisions for increasing energy or associated multiplicity, it is a priori not related to any macroscopic or bulk effects, much less to colour deconfinement. Vacuum excitation as well as exact strangeness conservation provide conventional monotonic descriptions of the enhancement observed in high energy nucleus-nucleus compared to p-p collisions. Before applying strangeness as a probe for deconfinement, it is therefore necessary to define a normal strangeness pattern, as seen in hadronic collisions and hence not related to QGP formation. This given, one could then check if sufficiently central nuclear collisions lead to any additional, non-montonic and hence unconventional effects.

The situation concerning transverse momentum broadening is quite similar. In nuclear collisions, expansion and flow of the produced medium are expected to lead to a broadening of the transverse momentum spectrum of secondary hadrons, and it has been suggested that the form of the broadening might reflect the transition to a QGP [13], flow effects [14] or the initial state scattering in the medium [56]. Indeed a mass-dependent broadening of $p_{T}$-spectra is observed e.g. in $\mathrm{Pb}-\mathrm{Pb}$ collisions; it increases with centrality (see Fig. 16). However, a very similar pattern, also increasing with particle mass, is observed in $\mathrm{p}-\overline{\mathrm{p}}$ collisions [59]; it increases with the associated multiplicity, as also shown in Fig. 16.
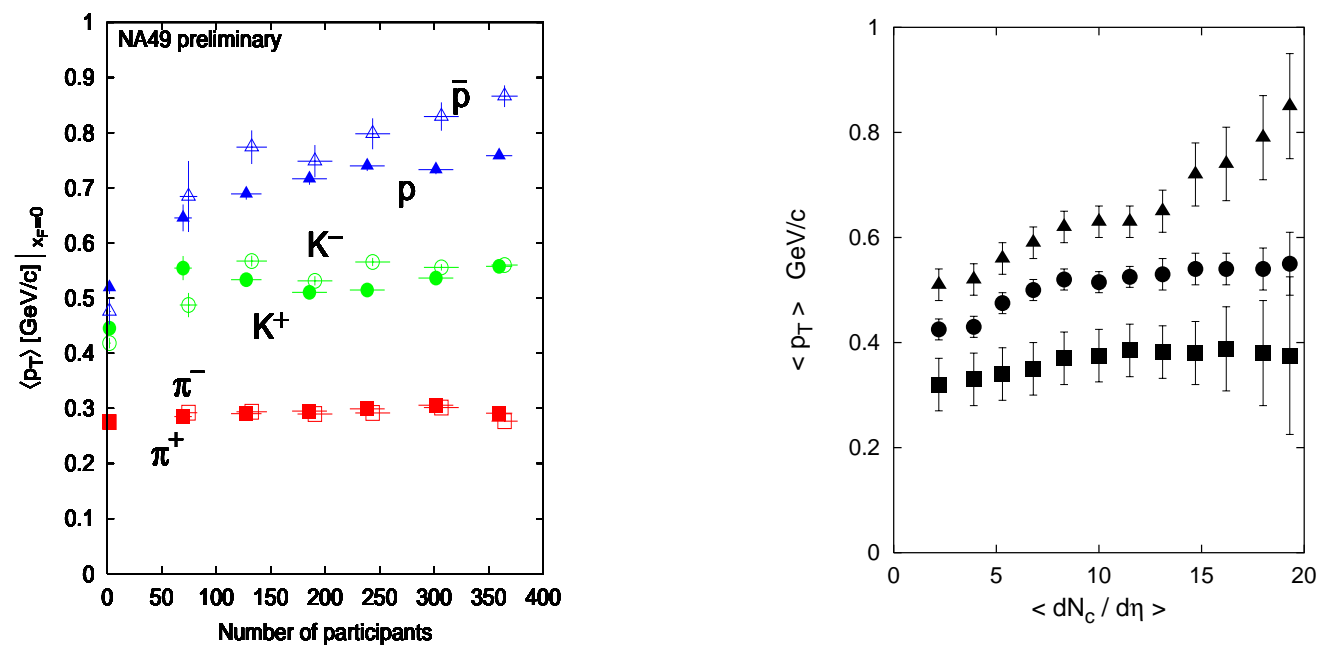

Figure 16: The average transverse momentum of different secondaries (left) in $\mathrm{Pb}-\mathrm{Pb}$ [61] and (right) p- $\overline{\mathrm{p}}$ collisions, same symbols [59].

In addition, there are other broadening effects known from $\mathrm{p}-\mathrm{p}$ and $\mathrm{p}-\mathrm{A}$ collisions. In hadron-hadron interactions, there is the so-called "seagull" effect, due to emission from a scattered primary nucleon; it leads to a increase of $p_{T}$ with $x_{F}$, until the kinematic 
limit requires a decrease. In p-A interactions, there is the Cronin effect [60, due to initial state scattering of the nucleon on its passage through the nucleus. Thus here as well the challenge is to define normal broadening, not related to deconfinement, as observed in p-p and $\mathrm{p}-\mathrm{A}$ collisions, and then check if nuclear collisions show more than this and if there is any kind of onset of anomalous behaviour. So far, this does not seem to be the case.

\section{Catching the Elusive}

Looking back to the beginning of the search, we note a definite progress in method: it is now clear how a systematic investigation must be carried out. Whatever probe is to be used, it must first be gauged in the absence of bulk matter, i.e., in p-p and/or $\mathrm{p}-\overline{\mathrm{p}}$ collisions. Following this, its behaviour must be gauged in normal nuclear matter as cold, confined medium. If possible, it should then be tested in a hot confined medium, e.g., in the collision of light nuclei. When all these tests clearly define the conventional behaviour of the probe, we can start to look for an onset of anomalous behaviour.

Where are we in this program with the different probes enumerated at the beginning of this appraisal?

- For charmonium suppression, normal behaviour is well-defined through p-A collisions and understood in terms of pre-resonance absorption in nuclear matter. This also accounts for all collisions up to central $\mathrm{S}-\mathrm{U}$ interactions. In $\mathrm{Pb}-\mathrm{Pb}$ collisions, there is a clear onset of anomalous behaviour once the collisions reach a certain centrality. The anomalour suppression pattern is in accord with the deconfinement predictions of stepwise $\chi_{c}$ and direct $J / \psi$ suppression, while it is in complete contradiction to all conventional hadronic scenarios.

$J / \psi$ suppression therefore constitutes the first and so far only observed unconventional sign. For its full understanding, however, a number of open questions still have to be answered.

- The dilepton enhancement in nucleus-nucleus collisions is specified in terms of a normal behaviour defined through hadronic decays in $\mathrm{p}-\mathrm{p}$ and $\mathrm{p}-\mathrm{A}$ collisions. In all nucleus-nucleus collisions studied, there are deviations; so far, no clear onset for these is observed. A conventional theoretical interpretation, based on resonance broadening in an interacting hadronic medium, appears to reproduce the effect. Interesting alternative explanations invoke changing hadron masses or thermal dileptons.

For more definite conclusions, also concerning the relation of low and intermediate mass dilepton enhancement, more precise data are necessary.

- A strangeness enhancement for increasing multiplicity or centrality is observed in all hadronic collisions, from p-p to A-A. There is so far no normal form and no observed onset. The effect may well provide for all cases a thermometer for the initial partonic environment; an increase of relative strangeness production between $\mathrm{p}-\mathrm{p}$ and $\mathrm{A}-\mathrm{A}$ is also predicted if strangeness conservation is taken into account exactly.

- A transverse momentum broadening is also observed quite generally in hadronic interactions, increasing with multiplicity in p-p, with $\mathrm{A}$ in $\mathrm{p}-\mathrm{A}$, and with centrality for A-A collisions. There are various normal mechanisms, such as initial and final state scattering, but so far there is neither a defined normal form nor any particular onset of the effect. 
A great present challenge, for theory as well as for experiment, is thus to define a normal form for strangeness production and transverse momentum behaviour; such a reference is a prerequisite in the search for unconventional signs.

What can these results and conclusions teach us for RHIC and LHC studies? First and foremost, I believe, they indicate that - barring the unexpected - higher collision energies are no substitute for a systematic study. As we have seen, strangeness enhancement and $p_{T}$-broadening occur in $\mathrm{p}-\mathrm{p} / \mathrm{p}-\overline{\mathrm{p}}$ collisions precisely at high energy. It is therefore necessary to study p-p and p-A collisions here as well, both in experiment and theory, before drawing any conclusions. If a given probe is not calibrated, it cannot provide convincing results.

Quite a number of different interesting experiments or experimental components will be devoted to the search for the QGP at RHIC and LHC. As in any scientific endeavor, we must be aware here that some results, even if they come from an extensive and welldone experimental study, may nevertheless simply be insensitive to deconfinement and QGP-formation. If we insist on finding the QGP everywhere, the scientific community at large may well doubt that we found it anywhere.

Last, but not least, it seems necessary that experiments are made to address fundamental physics questions; life is too short to refute all wrong models. Moreover, appearently dead models quite often sprout new, equally ugly heads, turning the battle against them into an everlasting hydradynamics 2 . The basic task will remain to first understand the conventional and then identify the unconventional signs.

\section{Acknowledgements}

The financial support by DFG contract Ka 1198/4-1, BMBF contract 06BI804/5 and GSI contract BISATT is gratefully acknowledged.

\section{References}

[1] M. Jacob and H. Satz, in Quark Matter Formation and Heavy Ion Collisions, World Scientific, Singapore 1982.

[2] P. Hut, Nucl. Phys. A418 (1984) 301c;

for a recent discussion of a similar danger, see

A. Dar, A. De Rujula and U. Heinz, Phys. Lett. B 470 (1999) 142;

W. Busza et al., hep-ph/9910333.

[3] E. V. Shuryak, Phys. Lett. 78 B (1978) 150

[4] K. Kajantie and H. I. Miettinen, Z. Phys. C 9 (1981) 341.

[5] J. D. Bjorken, Fermilab-Pub-82/59-THY (1982) and Erratum, unpublished.

[6] R. Baier, D. Schiff and B. G. Zakharov, Ann. Rev. Nucl. Part. Science, in press.

${ }^{2}$ The Hydra was a many-headed water serpent in Greek mythology. When one of its heads was cut off, two new heads would appear in its place. Hercules killed it by burning the neck after cutting off each head. 
[7] T. Matsui and H. Satz, Phys. Lett. 178B (1986) 416.

[8] D. Kharzeev and H. Satz, Phys. Lett. B 334 (1994) 155.

[9] R. Pisarski, Phys. Lett. B 110 (1982) 155.

[10] V. Bernard et al., Phys. Lett. 227 B (1989) 465;

G. E. Brown et al., Phys. Rev. C 43 (1991) 1881.

[11] J. Rafelski and B. Müller, Phys. Rev. Lett. 48 (1982) 1066;

J. Rafelski, Phys. Reports 88 (1982) 331.

[12] J. Rafelski, Phys. Lett. B 262 (1991) 153.

[13] L. Van Hove, Phys. Lett. B 118 (1982) 138.

[14] M. Kataja et al., Phys. Rev. D 34 (1986) 811 and 2755.

[15] For a survey, see L. Kluberg, Nucl. Phys. A 661 (1999) 300c.

[16] R. Rapp and E. Shuryak, Phys. Lett. B 473 (2000) 13.

[17] See F. Karsch, Nucl. Phys. B (Proc. Suppl.) 83-84 (2000) 14.

[18] N. Armesto et al., Phys. Rev. Lett. 77 (1996) 3736;

M. Nardi and H. Satz, Phys. Lett. B 442 (1998) 14;

H. Satz, Nucl. Phys. A 661 (1999) 104c.

[19] J. D. Bjorken, Phys. Rev. D27 (1983) 140.

[20] J. Bächler et al. (NA49), Nucl. Phys. A 661 (1999) 45c.

[21] M. C. Abreu et al. (NA50), Phys. Lett. B 410 (1997) 337, B 450 (1999) 456, and Phys. Lett. B 477 (2000) 28.

[22] D. Kharzeev, C. Lourenço, M. Nardi and H. Satz, Z. Phys. C 74 (1997) 307.

[23] M. Nardi, private communication.

[24] S. G. Matinyan and B. Müller, Phys. Rev. C 58 (1998) 2994;

K. L. Haglin, nucl-th/9907034 (1999);

Z. Lin and C. M. Ko, nucl-th/9912046 (1999);

K. Martins, D. Blaschke and E. Quack, Phys. Rev. C 51 (1995) 2723;

C.-Y. Wong, E. S. Swanson and T. Barnes, hep-ph/9912431 (1999).

[25] K. Redlich, H. Satz and G. M. Zinovjev, hep-ph/0003079; Europ. Phys. J. C, in press

[26] C. Baglin et al. (NA38), Phys. Lett. B 220 (1989) 471.

[27] J. Badier et al. (NA3), Z. Phys. C 20 (1983) 101.

[28] D. M. Alde et al. (E772), Phys. Rev. Lett. 66 (1991) 133. 
[29] C. Baglin et al. (NA38), Phys. Lett. B 446 (1999) 408.

[30] C. Gerschel and J. Hüfner, Z. Phys. C 56 (1992) 171.

[31] R. Vogt, Phys. Reports 310 (1999) 197.

[32] H. Satz, Rept. Prog. Phys. 63 (2000) 1511.

[33] For a survey, see R. V. Gavai et al., Int. J. Mod. Phys. A10 (1995) 3043.

[34] G. T. Bodwin, E. Braaten and G. P. Lepage, Phys. Rev. D 51 (1995) 1125;

E. Braaten and S. Fleming, Phys. Rev. Lett. 74 (1995) 3327.

[35] T. Affolder et al. (CDF), hep-ex/0004027;

E. Braaten et al., hep-ph/9911436.

[36] D. Kharzeev and H. Satz, Phys. Lett. B 366 (1996) 316.

[37] F. Karsch, M. T. Mehr and H. Satz, Z. Phys. C 37 (1988) 617.

[38] F. Karsch and H. Satz, Z. Phys. C 51 (1991) 209.

[39] S. Gupta and H. Satz, Phys. Lett. B 283 (1992) 439.

[40] C. Baglin et al. (NA38), Phys. Lett. B345 (1995) 617, B 449 (1999) 408, B 466 (1999) 128.

[41] H. Satz, Nucl. Phys. A 661 (1999) 104c.

[42] J.-P. Blaizot, Mai Dinh and J.-Y. Ollitrault, nucl-th/0007020.

[43] D. Kharzeev, M. Nardi and H. Satz, Phys. Lett. B 405 (1997) 14.

[44] R. L. Thews, M. Schroeter and J. Rafelski, hep-ph/0007323.

[45] P. Braun-Munzinger and J. Stachel, nucl-th/0007059.

[46] L. Ramello et al. (NA50), Nucl. Phys. A 638 (1998) 261.

[47] G. E. Brown and M. Rho, Phys. Rev. Lett. 66 (1991) 2720.

[48] R. Rapp and J. Wambach, hep-ph/9909229 (to appear in Adv. Nucl. Phys.).

[49] G. Agakiviev et al. (CERES), Nucl. Phys. A 610 (1996) 317c; Eur. Phys. J. C 4 (1998) 231; Nucl. Phys. A 638 (1998) 467c;

B. Lenkeit et al. (CERES), Nucl. Phys. A 661 (1999) 23c.

[50] M. C. Abreu et al. (NA38/50) Nucl. Phys. A 661 (1999) 538c, and Euro. Phys. J. C $13(2000) 69$.

[51] R. Hagedorn, Suppl. Nuovo Cimento 3 (1965) 147.

[52] F. Becattini, Z. Phys. C 69 (1996) 485;

F. Becattini and U. Heinz, Z. Phys. C 76 (1997) 269. 
[53] Yu. Dokshitzer, private communication.

[54] R. Hagedorn, CERN yellow report 71-12, 101 (1971);

R. Hagedorn and K. Redlich, Z. Phys. A 27 (1985) 541.

[55] S. Hamieh, K. Redlich and A. Tounsi, hep-ph/0006024.

[56] A. Leonidov, M. Nardi and H. Satz, Z. Phys. 74 (1997) 535.

[57] R. E. Ansorge et al. (UA5), Z. Phys. C 41 (1988) 179.

[58] C. Hoehne (NA49), Nucl. Phys. A 661 (1999) 485c.

[59] T. Alexopoulos et al., Phys. Rev. Lett. 64 (1990) 991.

[60] J. W. Cronin et al., Phys. Rev. D 11 (1975) 3105.

[61] G. Roland et al. (NA49), Nucl. Phys. A 638 (1998) 91c.

[62] F. Becattini et al. hep-ph/0002267;

J. Cleymans, private communication. 